\title{
Examples of Constrained Iterative Restoration of SPOT Panchromatic Images, with Prefiltering for Noise Reduction
}

By George Lemeshewsky

Open-File Report 94-251

U.S. Department of the Interior 


\title{
EXAMPLES OF CONSTRAINED ITERATIVE RESTORATION OF SPOT PANCHROMATIC IMAGES, WITH PREFILTERING FOR NOISE REDUCTION
}

\begin{abstract}
In this project, image blur was reduced in SPOT ${ }^{1} \mathrm{HRV}$ panchromatic band images by using iterative restoration techniques with modifications to suppress noise amplification. In the limit, as the iterations continue the restoration process converges to inverse filtering and thus is subject to excessive noise amplification. One method of reducing noise effects is to limit the iterations while achieving partial blur reduction; another is to suppress noise in the degraded image before restoration by preprocessing both the degraded image and the sensor system point spread function with a noise smoothing filter. A variation of the latter technique was used in this work. Instead of a smoothing filter, a smoothing and interpolating filter was applied, which also expanded the image size. Examples of iterative restoration, with a positive constraint to reduce ringing artifacts for both original size and enlarged size images, use simulated data and SPOT panchromatic spectral band, 10-m ground sample distance images. In this study, processing was terminated when the image, observed on a computer display monitor, was determined to have reasonable deblurring and tolerable noise amplification. In effect, this interactive technique falls between high spatial frequency enhancement for partial blur reduction and, in the limiting case, inverse filter restoration.
\end{abstract}

${ }^{1}$ Any use of trade, product, or firm names is for descriptive purposes only and does not imply endorsement by the U.S. Government. 


\section{INTRODUCTION}

The goal of image restoration is to recover the original scene from degraded observations using techniques oriented toward modeling the degradation, blur, and noise and applying an inverse procedure to obtain an approximation of the original scene (Katsaggelos, 1991). This report describes examples of image blur reduction by means of iterative inverse filtering for restoration. An advantage of this technique is that only a model of the blur degradation is needed; no noise model is required. This report follows a previous report (Lemeshewsky, 1993) that described blur reduction in SPOT panchromatic images and a technique to estimate the point spread function (PSF), which is the imaging system's impulse response or blur function. In the limit as the number of iterations approaches infinity, the process converges to inverse filtering and gives excessive noise amplification. In some cases, depending on the degree of blur and noise degradations, reasonable blur reduction can be achieved without objectionable noise levels by limiting the iterations (Lim, 1990).

Although noise amplification is a consequence of iterative inverse filtering, there are additional reported techniques (Schafer and others 1981; Kawata and Ichioka, 1980a) that mitigate noise effects. Two techniques are to apply constraints to the restoration solution or to first reduce the noise of the degraded image. The latter can be achieved by preprocessing the degraded image with a low-pass, noise smoothing filter; however, this additional smoothing (that is, blur) must be included in the degradation model. Schafer and others (1981) described the restoration of twodimensional signals, not images, by applying a smoothing and interpolating filter to both the degraded signal and the impulse response of the degradation model before restoration. Also, they found that constraints imposed on the iterative solution would reduce ringing and other artifacts. Application of these techniques to images is reported herein.

This report describes tests and gives examples of image blur reduction in which the degraded image was first preprocessed by a noise smoothing-interpolation (SI) technique to reduce noise amplification. Also the iterations were limited, and a positive constraint was applied to the iterative solution to suppress negative intensities caused by ringing and noise amplification. Restoration examples are given for simulated data and SPOT panchromatic band 10-m pixel images. The report is organized into five sections.

Section I briefly describes the noise amplification problem associated with inverse filter restoration. Section II describes the minor modification required in the iterative inverse filter process in order to use the SI technique for noise reduction. Section III gives examples of blur reduction for a simple, simulated image with additive noise when SI was used. Section IV gives examples of iterative restoration for blur reduction in SPOT 1, high-resolution visible, HRV-2, 10-m panchromatic band images at both the original and the two-times enlarged image size caused by smoothing-interpolation. Section $\mathrm{V}$ gives conclusions on these results. Note that when the iterations are limited, the result is a partially restored image. Subjectively, it may be an acceptable compromise between blur reduction and noise amplification.

\section{SECTION I. INVERSE FILTER RESTORATION}

The following discussion assumes a simple model of the imaging sensor system in which the degraded image $g(i, j)$, sensor system PSF $h(i, j)$, and original image $f(i, j)$ are assumed to be dis- 
crete. As defined in this study, the objective of restoration is to determine a discrete estimate of the original continuous image. The image is then observed as a continuous image on a computer device, typically at a magnified several times scale. Additional degradations from the display system were not considered in this study. The noise amplification problem common to inverse filtering is outlined next (Andrews, 1974).

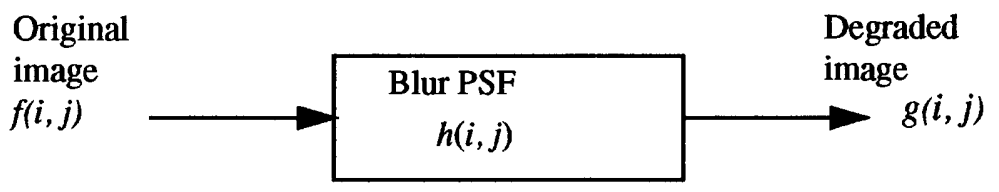

Figure 1. System model.

The system model of figure 1 can be expressed as

$$
g(i, j)=h(i, j) * f(i, j)
$$

where $g(i, j)$ is the degraded image, $h(i, j)$ is the PSF, the optical impulse response; * denotes convolution; and $f(i, j)$ is the original, undegraded, and assumed discrete image. The frequency domain equivalent of equation 1 is

$$
G\left(\omega_{1}, \omega_{2}\right)=H\left(\omega_{1}, \omega_{2}\right) F\left(\omega_{1}, \omega_{2}\right)
$$

where $F, G$, and $H$ denote the Fourier transform of $f, g$, and h, respectively; $\omega_{1}, \omega_{2}$ are spatial frequency variables; and $H\left(\omega_{1}, \omega_{2}\right)$ is the system transfer function. Inverse filter restoration of equation 2 to obtain $F\left(\omega_{1}, \omega_{2}\right)$ is

$$
F\left(\omega_{1}, \omega_{2}\right)=\left[1 / H\left(\omega_{1}, \omega_{2}\right)\right] G\left(\omega_{1}, \omega_{2}\right)
$$

where $1 / H\left(\omega_{1}, \omega_{2}\right)$ is the inverse filter for restoration. Including additive noise, $n(i, j)$, in the system model of figure 1 (equation 1) gives figure 2.

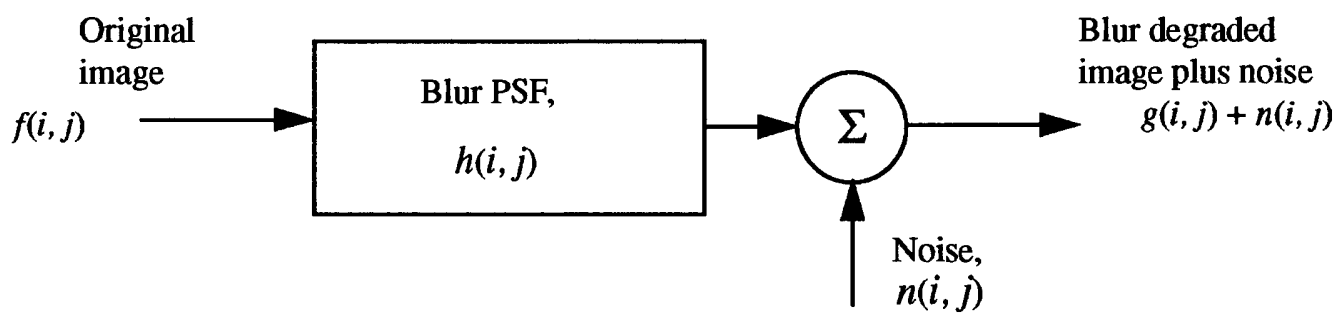

Figure 2. System model with additive noise degradation.

The model of figure 2 can be expressed as

$$
g(i, j)=h(i, j) * f(i, j)+n(i, j)
$$


or equivalently as

$$
G\left(\omega_{1}, \omega_{2}\right)=H\left(\omega_{1}, \omega_{2}\right) F\left(\omega_{1}, \omega_{2}\right)+N\left(\omega_{1}, \omega_{2}\right)
$$

where $N\left(\omega_{1}, \omega_{2}\right)$ is the Fourier transform of noise $n(i, j)$. Inverse filtering applied to equation 5 to give an estimate, $F^{\prime}\left(\omega_{1}, \omega_{2}\right)$, of the original $F\left(\omega_{1}, \omega_{2}\right)$ is

$$
\begin{aligned}
F^{\prime}\left(\omega_{1}, \omega_{2}\right) & =1 / H\left(\omega_{1}, \omega_{2}\right)\left[H\left(\omega_{1}, \omega_{2}\right) F\left(\omega_{1}, \omega_{2}\right)+N\left(\omega_{1}, \omega_{2}\right)\right] \\
& =F\left(\omega_{1}, \omega_{2}\right)+\left[1 / H\left(\omega_{1}, \omega_{2}\right)\right] N\left(\omega_{1}, \omega_{2}\right) .
\end{aligned}
$$

Here the estimate for undegraded $F\left(\omega_{1}, \omega_{2}\right)$ now includes noise, $N\left(\omega_{1}, \omega_{2}\right)$ multiplied, or amplified by inverse restoration filter $1 / H\left(\omega_{1}, \omega_{2}\right)$. Even if $N\left(\omega_{1}, \omega_{2}\right)$ is relatively low, small values of $H\left(\omega_{1}, \omega_{2}\right)$ can result in excessive noise amplification and unacceptable restoration.

\section{SECTION II. ITERATIVE INVERSE FILTER}

The iterative restoration equation, as described by Biemond and others (1990), is

$$
\begin{aligned}
& f_{k}(i, j)=\lambda g(i, j) \\
& f_{k+1}(i, j)=f_{k}(i, j)+\lambda\left[\left(g(i, j)-h(i, j) * f_{k}(i, j)\right]\right.
\end{aligned}
$$

where $f_{k=0}(i, j)=\lambda g(i, j)$ is the initial estimate for $f(i, j) ; g(i, j)$ is the degraded image; $h(i, j)$ is the PSF; * denotes convolution; $f_{k+1}(i, j)$ is the iterative approximation of $f(i, j)$, and $\lambda$ is an acceleration parameter. As described by $\operatorname{Lim}(1990)$, equation 7 can be expressed in the frequency domain as

$$
F_{k}\left(\omega_{1}, \omega_{2}\right)=\left[G\left(\omega_{1}, \omega_{2}\right) / H\left(\omega_{1}, \omega_{2}\right)\right]\left[1-\left(1-\lambda H\left(\omega_{1}, \omega_{2}\right)\right)^{k+1}\right] .
$$

Note that as $k$ approaches $\infty, F_{k}\left(\omega_{1}, \omega_{2}\right)$ approaches $G\left(\omega_{1}, \omega_{2}\right) / H\left(\omega_{1}, \omega_{2}\right)$, that is, restoration by inverse filtering, (equation 3 ), and provided that

$$
\left|1-\lambda H\left(\omega_{1}, \omega_{2}\right)\right|<1 .
$$

For example, if $H\left(\omega_{1}, \omega_{2}\right)$ is real and positive and $\lambda$ is real, then $0<\lambda<2$ (Schafer and others, 1981). In this study, $\lambda \leq 1.0$ was used; it satisfies the conditions of equation 9 .

After normalized spatial frequency variable $u$ is substituted (where $u=1 / 2 \Delta x$ and $\Delta x$ is the normalized sample distance, 1$)$ for $\left(\omega_{1}, \omega_{2}\right)$ in equation 8, figures 3(a) and 3(b) show, respectively, plots of the iterative filter term of equation 8 , that is, $[1 / H(u)]\left[1-(1-\lambda H(u))^{k+1}\right]$, and the restored transfer function after restoration, equation 8 , as a function of iteration count, $k$. In this example the spatial frequency domain degradation, $H(u)$, was Gaussian blur, and $\lambda$ was equal to 1 . Note that the degree of high-pass filtering of figure 3(a) can be controlled experimentally by iterations $k$. 

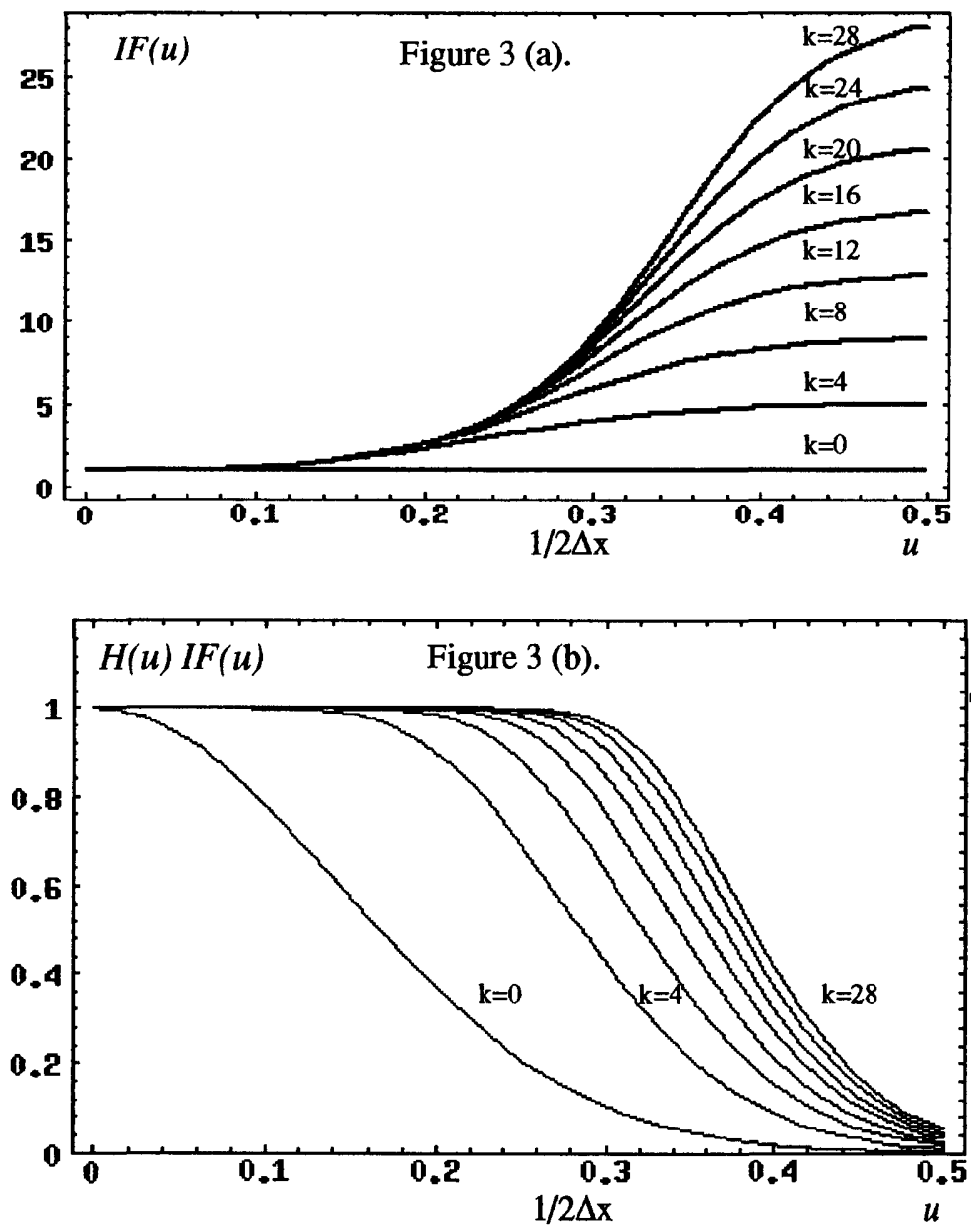

Figure 3(a). This illustration shows the itertive filter term, $I F(u)$, of equation 8; that is, $I F(u)=[1 / H(u)]\left[1-(1-\lambda H(u))^{k+1}\right]$ for $k=0$-to- 28 and $\lambda=1.0$. Spatial frequency $u$ $=1 / 2 \Delta x$ where $\Delta x$ equals 1 is the sample distance. The Nyquist frequency is thus 0.5 . These plots show the frequency correction, per iterations $k$, that would be applied when the degradation transfer function (TF), $H(u)$, as shown in figure 3(b) is Gaussian.

Figure 3(b). This illustration shows the restored TF; that is, equation 8 , or $H(u) I F(u)$ for $k=0$-to- 28 and where $I F(u)$ was plotted in figure $3 \mathrm{a}$. The Gaussian degradation TF, $H(u)$ is that for $k=0$.

In the limit as $\mathrm{k}$ approaches $\infty$, the iterative process (equation 8 ) approaches the inverse filter, which can cause excessive noise amplification and hence unacceptable image quality. Several techniques (Biemond and others, 1990; Kawata and Ichioka, 1980; Schafer and others, 1981) to control noise artifacts include limiting the iterations, imposing constraints on the process, and reducing the noise in the degraded image before restoration. All were applied in this study. Note that when the iterations are limited, the result is not the same as inverse filter restoration, but the reduced blur may be acceptable (Lim, 1990).

\section{SECTION III. NOISE PREFILTERING}

When there are additive noise degradations (for example, due to the sensor systems electronic components or quantization effects), the quality of images restored by inverse filtering can be unacceptable. One approach to lessen noise amplification is first to apply a low-pass, noise-reducing spatial filter to the degraded image (Kawata and Ichioka, 1980). Restoration is possible if the imaging system model is modified to include the additional degradation due to noise smoothing. This modification is described next.

Let $s(i, j)$ be a low-pass, noise-smoothing filter, applied to both degraded image $g(i, j)$ and PSF 
$h(i, j)$. Then

$$
\begin{aligned}
& g^{\prime}(i, j)=g(i, j) * s(i, j) \\
& h^{\prime}(i, j)=h(i, j) * s(i, j)
\end{aligned}
$$

where $g^{\prime}(i, j)$ is now a new degraded image, additionally blurred by $s(i, j)$, and $h^{\prime}(i, j)$ is the new PSF of the overall degradation; $*$ denotes convolution. The modified imaging system that includes the degradation due to filter $s(i, j)$ model is shown in figure 4 .

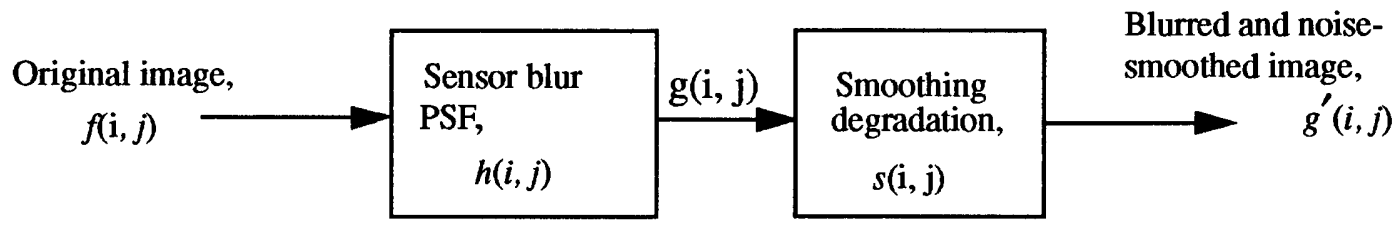

Figure 4. System model with noise-smoothing filter degradation, $s(i, j)$.

Iterative restoration for the model of figure 4 is found by replacing $g(i, j)$ and $h(i, j)$ of equation 7 with $g^{\prime}(i, j)$ and $h^{\prime}(i, j)$ of equations 10 and 11 , respectively. This gives

$$
\begin{aligned}
& f_{k}(i, j)=\lambda g^{\prime}(i, j) \\
& f_{k+1}(i, j)=f_{k}(i, j)+\lambda\left[g^{\prime}(i, j)-h^{\prime}(i, j) * f_{k}(i, j)\right]
\end{aligned}
$$

where $f_{k=0}(i, j)=\lambda g^{\prime}(i, j)$ is the initial estimate for image $f(i, j)$ and $h^{\prime}(i, j)$ is the overall blur degradation given by equation $11 ; g^{\prime}(i, j)$ is the degraded image, $g(i, j)$, after convolution with noisesmoothing filter $s(i, j)$ (that is, equation 10$)$.

Filter $s(i, j)$ need not be only a smoothing process. Schafer and others (1981) described application of a smoothing and interpolating filter with iterative restoration of blur and noise-degraded impulse-like signals. This study describes preliminary tests of the restoration of images, with preprocessing by means of a smoothing-interpolating filter to reduce noise and also to expand the image size.

For restoration of impulse-like signals with low background levels, Schafer and others (1981), and Biemond and others (1990) reported a reduction in ringing artifacts when a positive constraint was applied to the iterative solution. This constraint clips negative values to zero. In this study, both a positive and a maximum constraint were applied to equation 7 or 12 , as follows:

$$
f_{k+1}(i, j)=P\left[f_{k+1}(i, j)\right]
$$

where constraint operator $P[]$ was

$$
P[x]=\left\{\begin{array}{rlr}
0 & \text { when } & x=0 \\
x & \text { when } & 0<x<255 \\
255 & \text { when } & x \geq 255
\end{array}\right.
$$


Positive values were clipped to 255 in order to display image results with 8-bits per pixel. For the image sample of this study, image features such as roads have relatively high gray levels in a low background. Therefore, the positive constraint of equation 14 (that is, clipping negative values to zero) was applied in an attempt to reduce ringing artifacts that produce negative gray values.

\section{SECTION IV. EXAMPLES - SIMULATED DATA}

Two experiments were conducted to demonstrate iterative restoration, with SI to reduce noise levels in the degraded image before the iterative restoration. The PSF, $h(i, j)$, for the following tests was that previously estimated for the SPOT panchromatic sensor system in Lemeshewsky (1993). Simulated, undegraded image data, $f(i, j)$, were derived from a computer-generated profile (row) of 128 intensity values; these values were merely repeated row-by-row to make a simple 128- by 128-pixel image. The degraded image, $g(i, j)$, was simulated by convolving $f(i, j)$ with $\mathrm{h}(i, j)$, equation 1. In one test, the image included additive, zero mean, white Gaussian noise (Kay, 1988).

For tests in the following section, without SI the restoration equation was

$$
\begin{aligned}
& f_{k}(i, j)=\lambda g(i, j) \\
& f_{k+1}(i, j)=P\left[f_{k}(i, j)+\lambda\left[g(i, j)-h(i, j) * f_{k}(i, j)\right]\right]
\end{aligned}
$$

For the tests in this section, with SI the restoration equation was

$$
\begin{aligned}
& f_{k}(i, j)=\lambda g^{\prime}(i, j) \\
& f_{k+1}(i, j)=P\left[f_{k}(i, j)+\lambda\left[g^{\prime}(i, j)-h^{\prime}(i, j) * f_{k}(i, j)\right]\right]
\end{aligned}
$$

where smoothed and interpolated $g^{\prime}(i, j), h^{\prime}(i, j)$ were that of equations 10 and 11 respectively, and $\mathrm{P}[\mathrm{]}$ was equation 14. Acceleration parameter $\lambda$ was equal to 1.0.

In this work $s(i, j)$, the filter for SI, was implemented by first placing a row (column) of zero values between every row (column) of degraded image $g(i, j)$ and PSF $h(i, j)$ so as to double the spatial size. The result was then convolved with a separable smoothing filter $s(i, j)=s_{1}(i) s_{2}(\mathrm{j})$, where $s_{1}(i)=s_{2}(j)=0.05,0.25,0.40,0.25,0.05$ (see Lim, 1990, for details). For the following tests, the number of iterations, $k$, was limited so that viewed subjectively, the partially restored image, $f_{k+1}(i, j)$, evidenced reasonable deblurring without excessive noise amplification.

For simulated data without noise, figure 5 shows restoration by the SI technique. It consists of a series of intensity plots from a central row of the simple, simulated, undegraded image shown in figure 6.

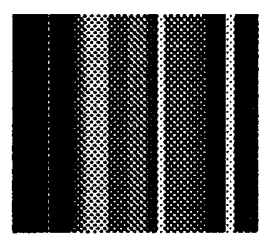

Figure 6. Simulated test image. 
A plot from a row of the undegraded (that is, without blur) image is shown in figure 5(a). Figure 5(b) shows the blurred profile after convolving the image of figure 6 with PSF $h(i, j)$. Figure 5(c) shows figure 5(b) plot after SI to a two-times larger size. Figures 5 (b) and (c) are, respectively, $\mathrm{g}(i, j)=h(i, j) * f(i, j)$ (equation 1) and $g^{\prime}(i, j)=s(i, j) * g(i, j)$ (equation 10).

For comparison, figure 5(d) shows the undegraded profile of figure 5(a) after SI to two-times larger size. Figure 5(e) is the result of 15 iterations of constrained restoration, equation 16, applied to figure 5(c). A reduction in blur is evident when figures 5(a) and 5(e) are compared. Table 1 summarizes the data of figure 5 .

Table 1. Summary of plots shown in figure 5

\begin{tabular}{|l|l|l|}
\hline \multicolumn{1}{|c|}{ Plot } & \multicolumn{1}{c|}{ Description } & \multicolumn{1}{c|}{ Processing } \\
\hline \hline 5 (a) & Undegraded input & $f(i, j)$ \\
\hline 5 (b) & Blurred [ 5 (a) ] & $h(i, j) * f(i, j)$ \\
\hline 5 (c) & SI [ 5(b) ] & $s(i, j) * h(i, j) * f(i, j)$ \\
\hline 5 (d) & SI [ 5 (a) ] & $s(i, j) * f(i, j)$ \\
\hline 5 (e) & Restored [ 5 (c) ] & Equation 16; 15 iterations \\
\hline
\end{tabular}

Figure 7 shows a series of plots for conditions similar to those of figure 5, except that zero mean, white Gaussian noise, $n(i, j)$, with variance $=2.0$, was added to the blurred image; figure 5(b) showed a row of the blurred image. Figure 7(b) is figure 5(b) plus noise. A comparison of plots $7(a)$ and 7(e) shows blur reduction, noise amplification, and ringing near abrupt transitions. Table 2 summarizes the data of figure 7. Reduced blur in the presence of noise can be seen by comparing plots 7(a) and 7(e). Results without noise were given in figure 5(a) and (b).

Table 2. Summary of plots shown in figure 7

\begin{tabular}{|l|l|l|}
\hline Plot & \multicolumn{1}{|c|}{ Description } & \multicolumn{1}{c|}{ Processing } \\
\hline \hline 7 (a) & Undegraded input & $f(i, j)$ \\
\hline 7 (b) & Blurred [ 7 (a) $]+\mathrm{n}(\mathrm{i}, \mathrm{j})$ & $h(i, j) * f(i, j)+n(i, j)$ \\
\hline 7 (c) & SI [ 7 (b) ] & $s(i, j) *\{h(i, j) * f(i, j)+n(i, j)\}$ \\
\hline 7 (d) & SI [ 7 (a) $]$ & $s(i, j) * f(i, j)$ \\
\hline 7 (e) & Restored [ 7 (c) $]$ & Equation $16 ; 15$ iterations \\
\hline
\end{tabular}

\section{SECTION V. EXAMPLES - SPOT IMAGES}

In the following examples, image data before restoration were SPOT -1 HRV -2 , preprocessing level 1A, 10-m pixel panchromatic band images (@ 1988, Licensed by SPOT Image Corporation, Reston, Virginia). Level 1A images are raw data, processed only for detector normalization (SPOT Image Corp., 1988). To improve the effectiveness of the positivity constraint (equation 14) in suppressing noise and ringing artifacts that show up as negative intensities for $f_{k}(i, j)$, the back- 
ground intensity of the raw image was reduced by linear-scaling the image intensities so that the minimum gray level, 24, and the mean plus six standard deviations, 180, became 0 and 255 , respectively.

In figure 8, image (a) is a 256- by 256-pixel sample from a raw SPOT image, processing level 1A; image (b) is the result after 8 iterations of positivity constrained iterative restoration deblurring, equation 15. Image (c) is image (b) enlarged two times by cubic interpolation. The number of iterations were simply based on a subjective evaluation of the reduced blur and increased noise.

Figure 9 shows the image of figure 5(a) enlarged to twice its original size by either SI (fig. 9(a)), or cubic interpolation (fig. 9(b)). They are given for comparison with the following images.

Figure 10(a) shows the result of partial, iterative restoration using SI of equation 16, iterations, applied to figure 9(a), the noise smoothed-interpolated raw image that was given in figure 8 (a).

Also shown for comparison with the previous SPOT image examples is figure 10(b), an image derived from a digitally scanned (at 2-m sample distance) color-infrared photograph (Dickson, 1989). It is the green color-separate in intensity units, not film density units, and cubic interpolated to $5 \mathrm{~m}$. The spectral response of this image (Eastman Kodak, 1992), approximately 550 to $675 \mathrm{~nm}$, is roughly comparable to the 510 to $730 \mathrm{~nm}$ response of the SPOT panchromatic sensor (SPOT, 1988).

Figure 11 shows plots of two types of errors versus iterations, $k$, for SI restoration of the raw SPOT image of figure 9(a). For the data of plot 1, RMS restoration error defined herein as $\left[g^{\prime}(i, j)-h^{\prime}(i, j) * f_{k}(i, j)\right]$ was measured on the entire image, less a 20-pixel margin to exclude border effects of the restoration process, as implemented. Plot 2, a rough measure of RMS noise error defined as $f_{k}(i, j)-g^{\prime}(i, j)$, was measured in a 19- by 19-pixel area of relatively constant and low (approximately 15) gray level. It is a measure of the difference between the expanded, restored image and the raw image after SI. Observe that for $k$ larger than about 10, there is little change in restoration error (plot 1); thus a possible choice for $k$ might be 10 . Note that the noise error, plot 2 , continues to increase with $k$; this illustrates the noise amplification problem of iterative inverse filter restoration.

Figure 12 shows plots of frequency spectra before and after partial restoration of the original-size image and of the double-size image from SI. These data show the log magnitude of the discrete Fourier transform, DFT, of a 256- by 256-pixel subregion, beginning at row 20, column 20, of the SI enlarged 512- by 512-pixel images. The two-dimensional (2-D) DFT was implemented from the one-dimensional (1-D) fast Fourier transform given in Gonzalez and Wintz (1977). Note that the normalized spatial frequency of 0.5 is the Nyquist frequency of the enlarged images; thus 0.25 is the Nyquist frequency of the original-size image. In figure 12, one effect after restoration is an increase in the amplitudes of frequency spectra, when $u$ or $v$ is less than 0.25 ; these data also show roughly similar results with or without SI.

Before the DFT calculation, the images were multiplied by a two-dimensional window function to reduce spectral leakage effects ( $\mathrm{Lim}, 1990)$. It was the product of two, one-dimensional cosine squared, or Hann, window functions; see Harris (1978) for additional details on DFT windowing 
techniques.

Figure 12 contains 1-D plots from the 2-D DFT, and figure 13 shows log magnitude of the corresponding 2-D DFT. Spectral components for spatial frequencies $u$ or $v>0.25$ may be due to noise or interpolation artifacts and are subject to further study.

\section{SECTION VI. CONCLUSIONS}

Preliminary examples were given to illustrate partial image restoration by an iterative technique, where the raw image was preprocessed first and also enlarged to twice its original size with a noise smoothing-interpolating filter. Also there were examples of partial restoration of the original-size image followed by cubic interpolation to twice that size. For these two conditions, the simple comparative examples are similar. To control noise amplification, the iterations were limited and intermediate results constrained to positive values. Although the ability of the positive constraint to suppress noise and ringing artifacts was not evaluated in this study, it is believed to be beneficial when there are low background intensities.

Although the blur reduction and related noise amplification of this iterative, partial restoration technique was subjective, its simplicity is attractive, especially when it is used with an interactive display system. Only a model of the degradation is required; no noise model or inverse restoration model is needed. This technique is not equivalent to restoration, but the experimenter can stop the processing when blur reduction and the inevitable noise amplification are acceptable. This technique might be useful on workstations as an interactive tool for partial blur reduction.

\section{REFERENCES}

Andrews, H.C., 1974, Digital image restoration - A survey: Computer, v. 7, no. 5, p. 36-45.

Biemond, J., Lagendijk, R.L., and Mersereau, R.M., 1990, Iterative methods for image deblurring: Proceedings of the IEEE, v. 78, no. 5, p. 856-882.

Dickson, R.A., 1989, Products available from NAPP imagery, v. 1, Technical Papers, ASPRS/ACSM Annual Convention, 4/2-7/89, Baltimore, Md.

Eastman Kodak Company, 1992, Aerial data: Rochester, N.Y., Eastman Kodak Company, Infrared film specifications AS-69. in Kodak Data for Aerial Photography, 1992, sixth edition, Kodak Publication no. AS-29, Eastman Kodak Company.

Gonzalez, H.C., and Wintz, P., 1977, Image transforms: Digital Image Processing, Boston, AddisonWesley, chap. 3, p. 36-114.

Harris, F.J., 1978, On the use of windows for harmonic analysis with the discrete Fourier transform: Proceedings of the IEEE, v. 66, no. 1, p. 51-83.

Katsaggelos, A.K., 1991, Introduction in Katsaggelos, A.K., ed., Digital image restoration: New York, Springer-Verlag., chap. 1, p. 1-20.

Kawata, S. and Ichioka, Y., 1980a, Iterative image restoration for linearly degraded images, I. Basis: Journal of the Optical Society of America, v. 70, no. 7, p. 762-768.

Kawata, S. and Ichioka, Y., 1980b, Iterative image restoration for linearly degraded images, II. Reblurring procedure: Journal of the Optical Society of America, v. 70, no. 7, p. 768-772. 
Kay, S.M., 1988, Computer program to generate real white Gaussian noise, in Modern spectral estimation: Englewood Cliffs, New Jersey, Prentice Hall, Appendix 5a, p. 145-147.

Lemeshewsky, G.P., 1993, Iterative restoration deblurring of SPOT panchromatic images, U.S. Geologicial Survey Open-File Report 93-353, 22 p.

Lim, J.S., 1990, Two-dimensional signal and image processing: Englewood Cliffs, N. J., Prentice-Hall, $694 \mathrm{p}$.

Schafer, R.W., Mersereau, R.M., and Richards, M.A., 1981, Constrained iterative restoration algorithms: Proceedings of the IEEE, v. 69, no. 4, p. 432-450.

SPOT Image Corporation, 1988, SPOT user's handbook: Reston, Va., SPOT Image Corporation, v. 1, 2. 


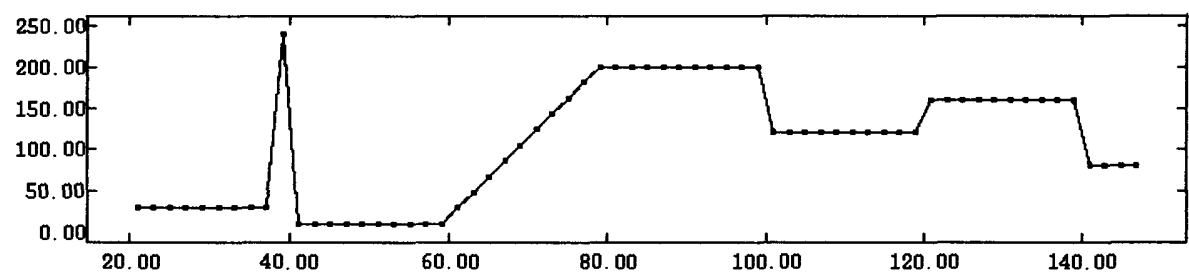

(a) Original

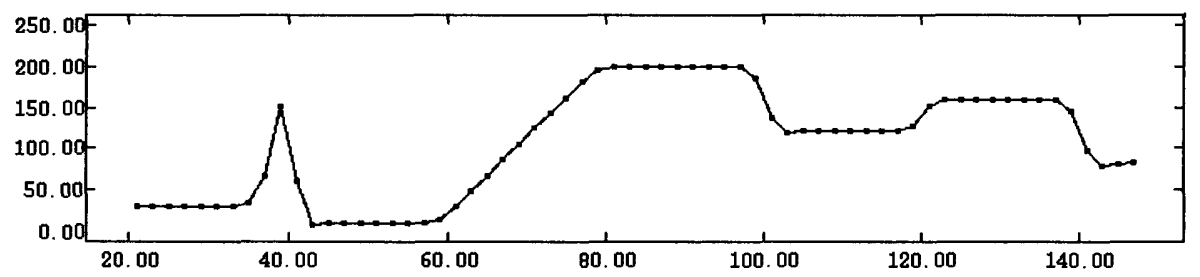

(b) Blurred (a)

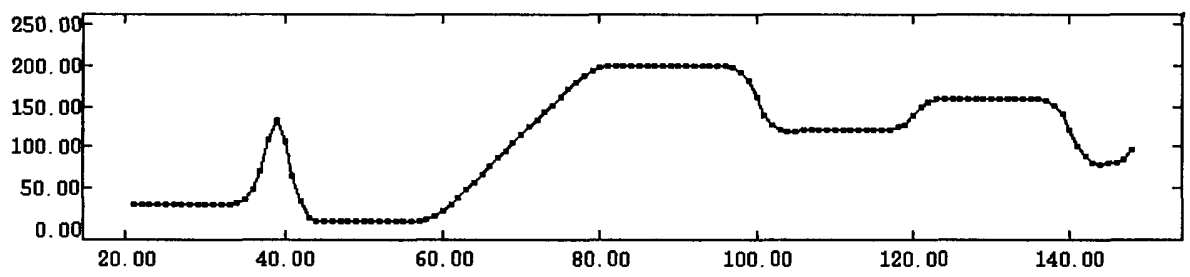

(c) Blurred data of (b) after smoothing-interpolation.

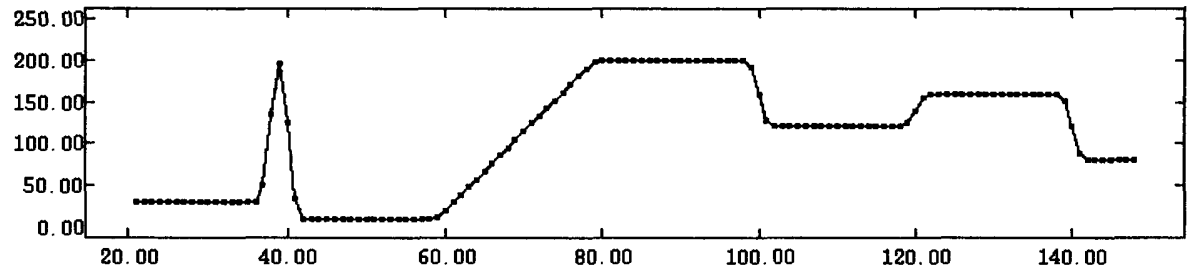

(d) Original of (a) after smoothing-interpolation

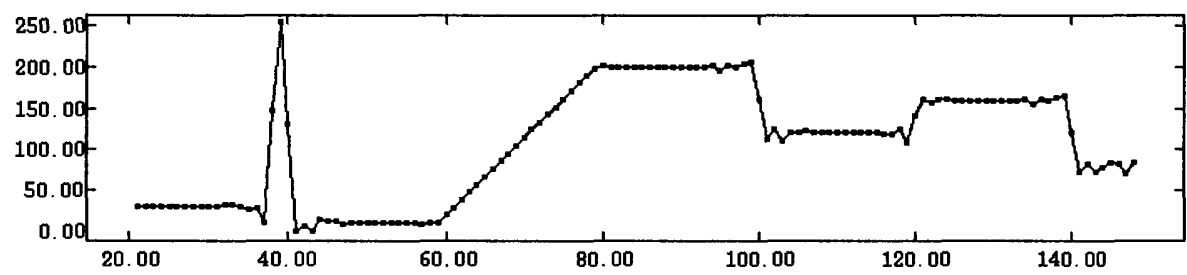

(e) Restoration of (c)

Figure 5. Restoration results of simulated, noiseless image after interpolation to twice the original size.

(a) Row of original image of figure 6 with samples shown on expanded scale. (b) Blurred data made by convolving (*) the original simulated image with the sensor point spread function (PSF), per equation 1. (c) Blurred and interpolated (twice size) data of (b) made by convolving (b) with the smoothing-interpolation filter, $\mathbf{s}(\mathrm{i}, \mathrm{j})$, per equation 10. (d) original data of (a) after interpolation to twice size by $s(i, j)$. (e) Result of restoration applied to (c) after 15 iterations, per equation 12. Here the combined degradation PSF was [sensor PSF] * [s(i,j)], per equation 11. Comparing (e) with (d) and (a) shows that the sensor-induced blur and additional degradation of the smoothing-interpolation process have been reduced by the restoration process. 


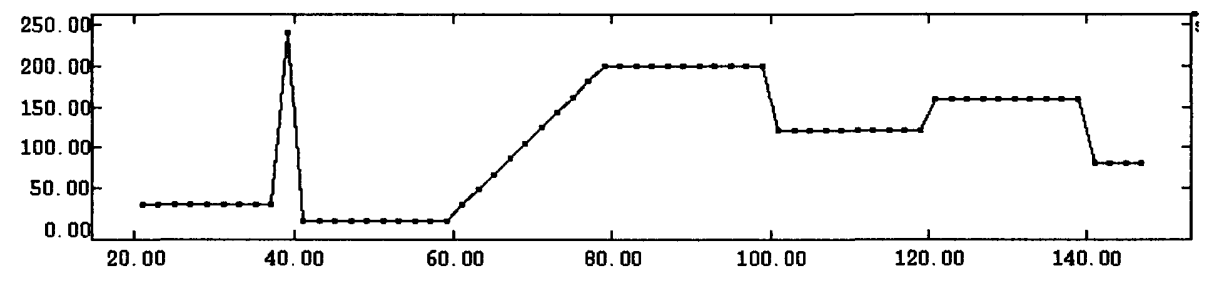

(a) Original

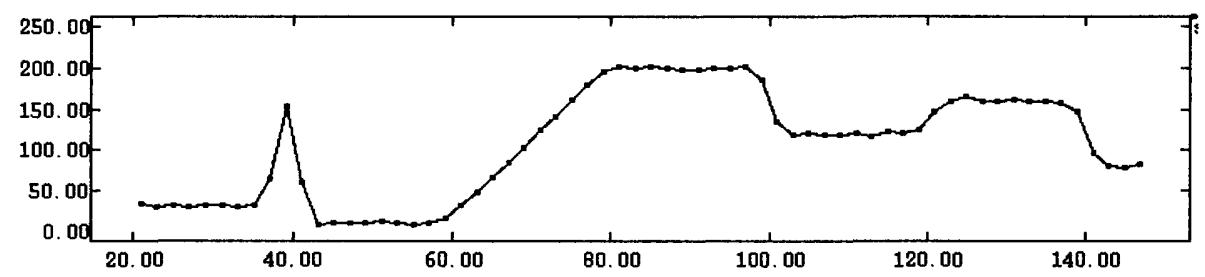

(b) Blurred (a) plus noise

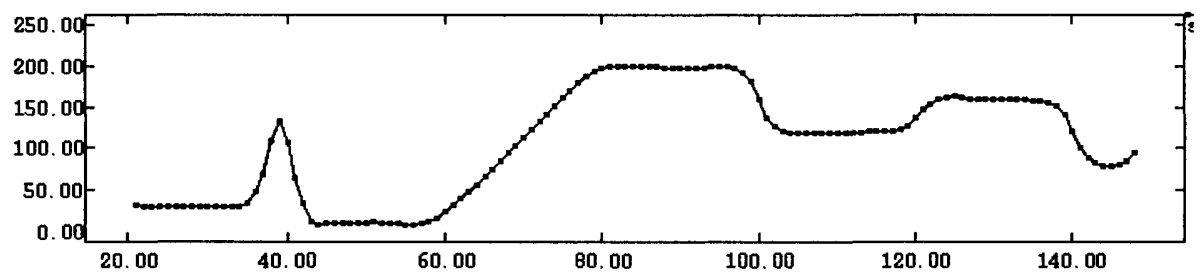

(c) Data of (b) after smoothing-interpolation

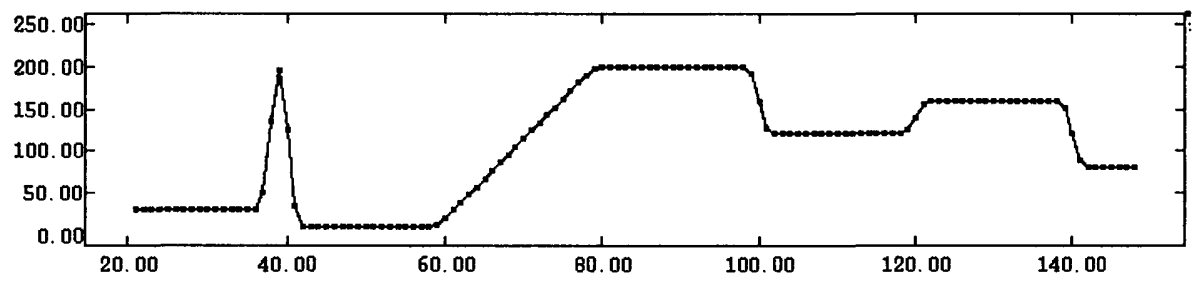

(d) Original of (a) after smoothing-interpolation

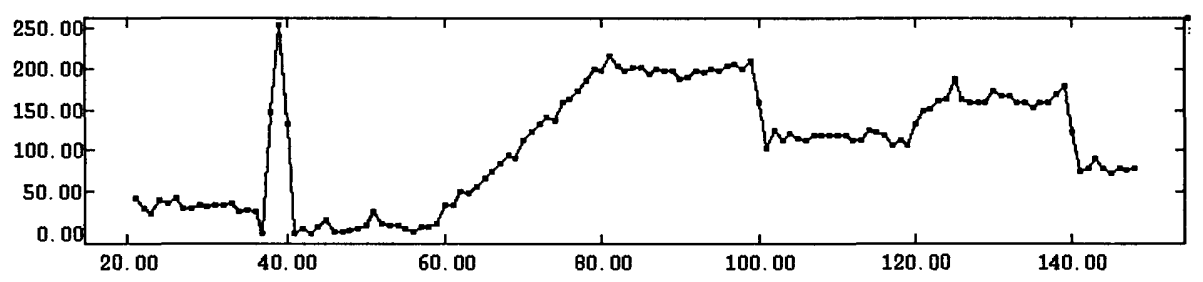

(e) Restoration of (c)

Figure 7. Restoration results of simulated image with noise, after interpolation to twice the original size.

(a) Row of original image of figure 6 with samples shown on expanded scale. (b) Blurred data, with additive Gaussian noise (variance $=2.0$ ) made by first convolving $(*)$ the original simulated image with the sensor point spread function (PSF), then adding noise, per equation 1. (c) Blurred and interpolated (twice size) data of (b) made by convolving (b) with the smoothing-interpolation, (SI), filter, $s(i, j)$, per equation 10. (d) original data of (a) after interpolation to twice size by SI. (e) Result of restoration applied to (c) after 15 iterations, per equation 12 . As in figure 5, the combined degradation PSF was [sensor PSF] * [s(i, j)], per equation 11. Comparing (e) with (d) and (a) shows that the sensor induced blur and additional degradation of the smoothing-interpolation process have been reduced by the restoration process; however, noise amplification is apparent. 
(a)

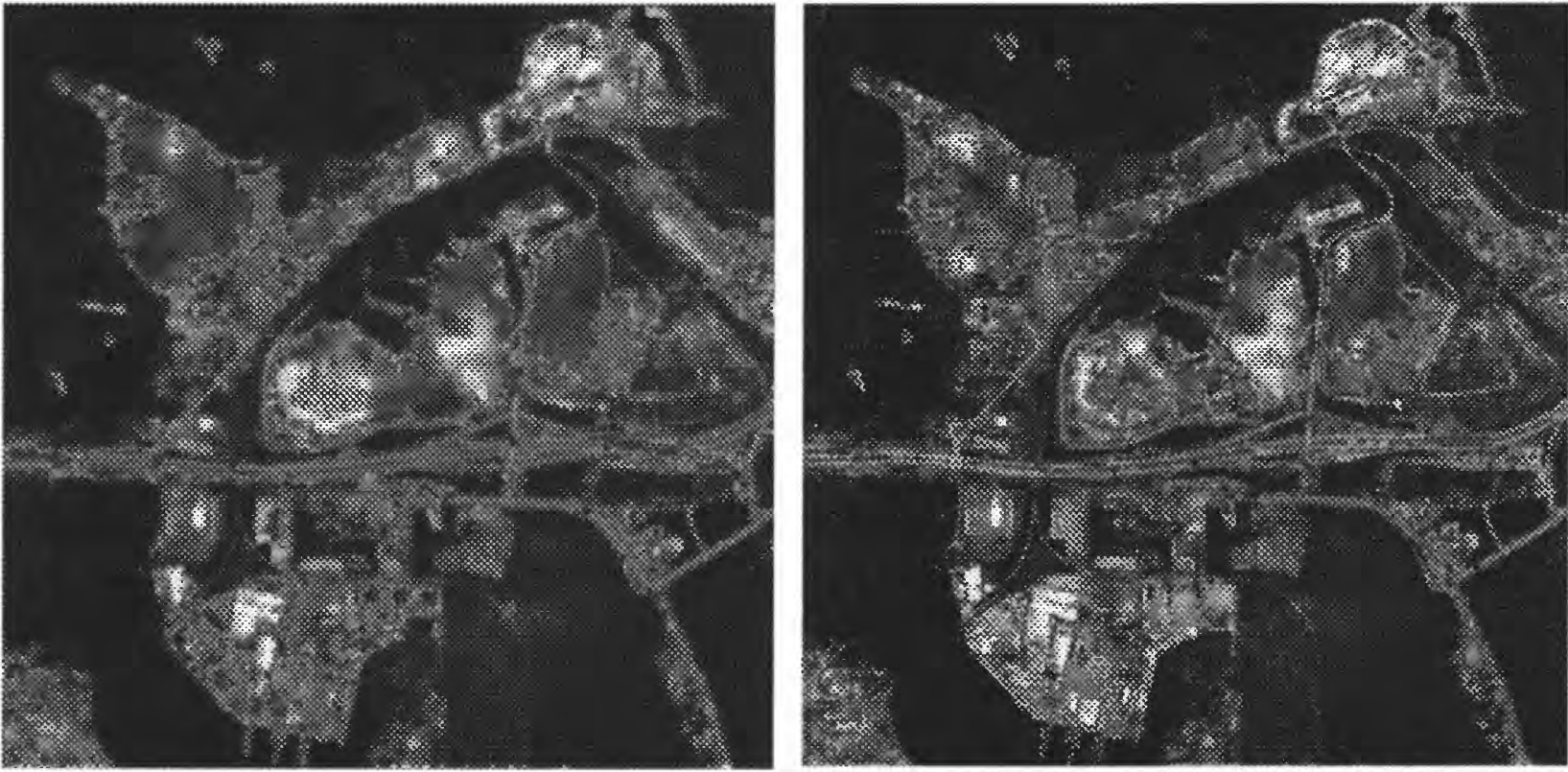

(b)

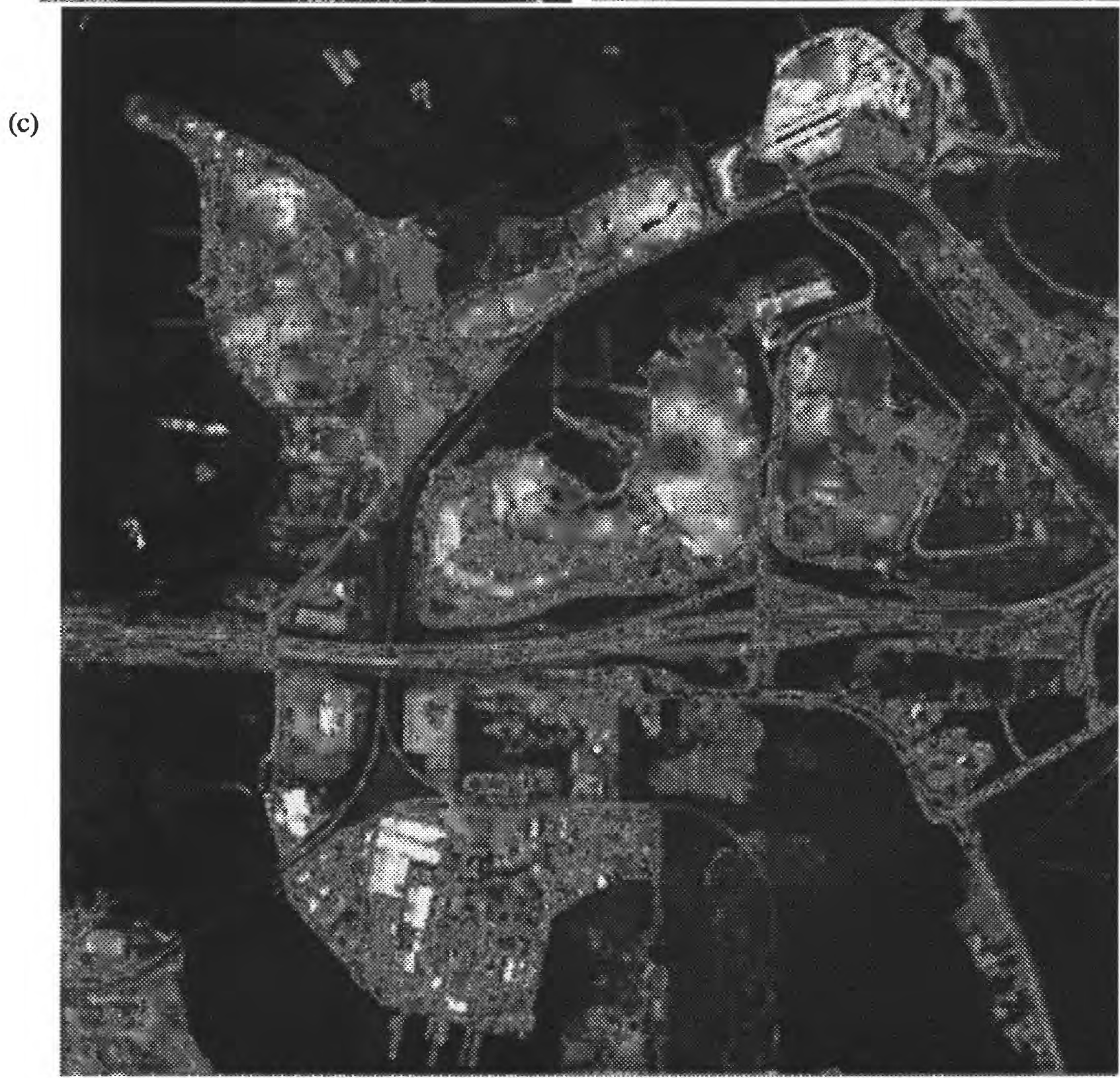

Figure 8. (a) Raw SPOT panchromatic image sample, 256 x 256 pixels, (ㄷ 1988, licensed by SPOT Image Corporation, Reston, VA). (b) Restoration (8 iterations) of (a). (c) Image (b) enlarged two times by cubic interpolation. 


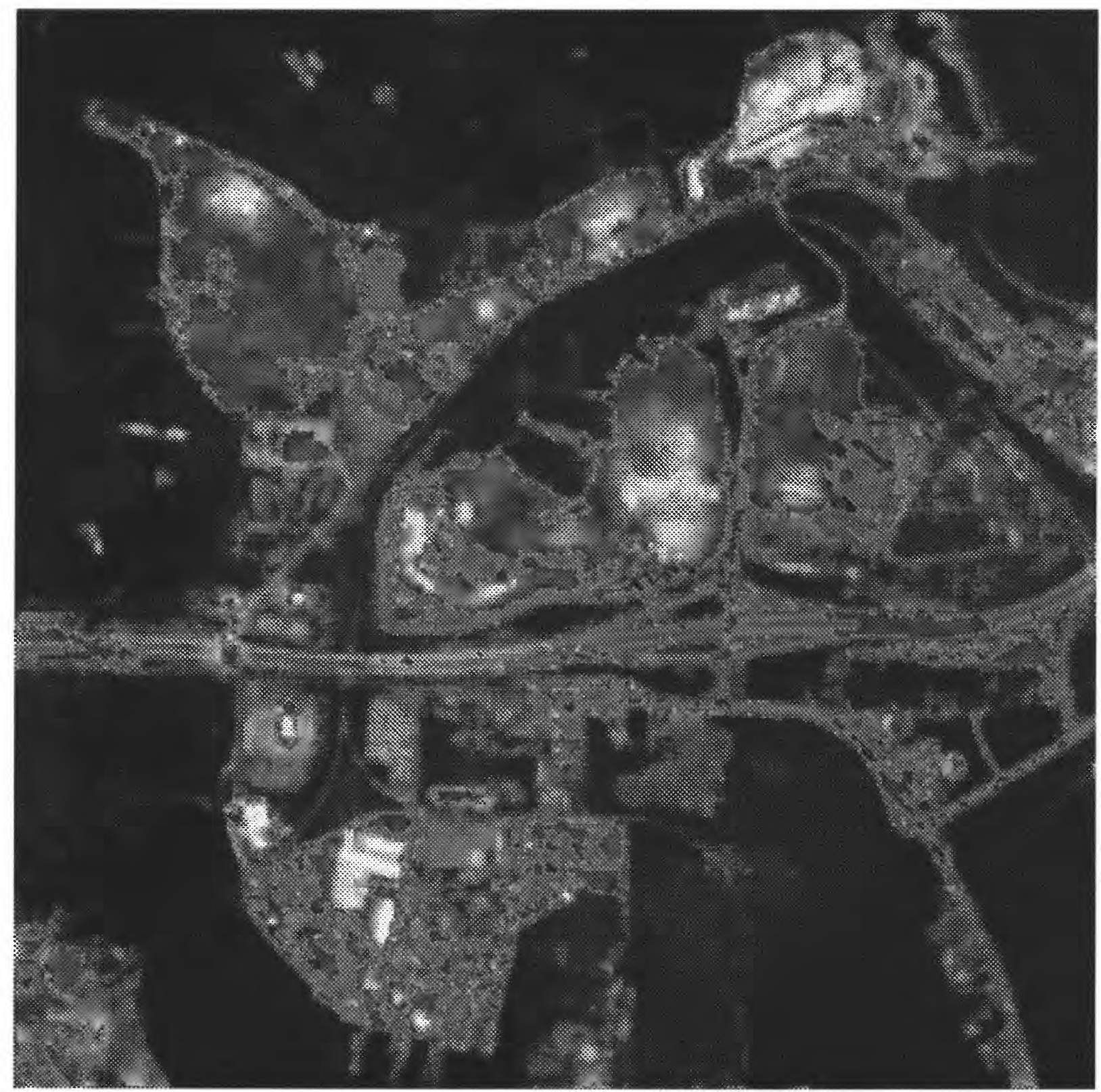

Figure 9 (a). Raw image of figure 8 (a) enlarged two times by convolution with smoothing-interpolation filter described in Section III. 


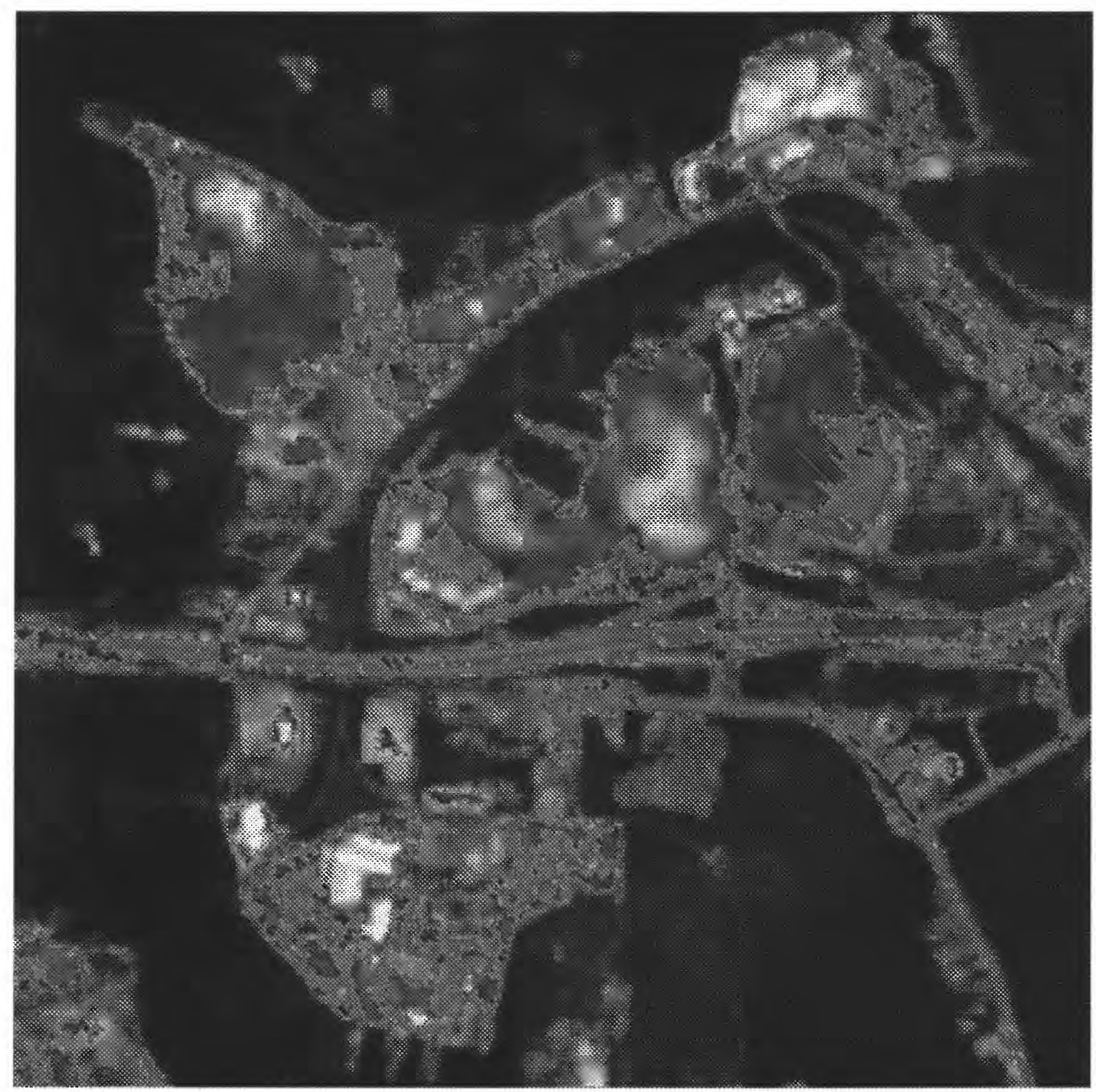

Figure 9 (b). Raw image of figure 8 (a) enlarged two times by cubic interpolation. 


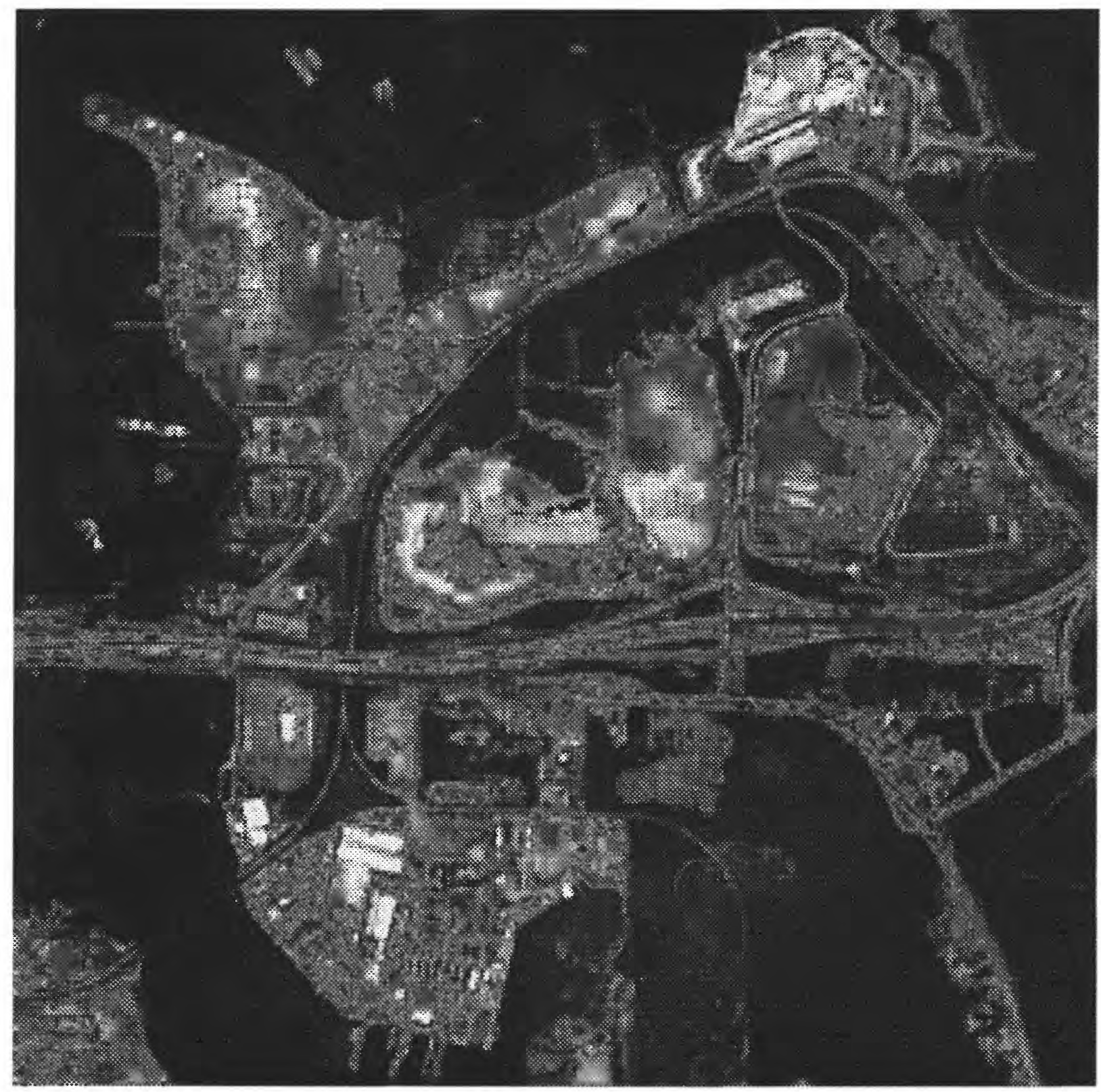

Figure 10 (a). Result of restoration (equation 16), 13 iterations, applied to the two-times enlarged, (by smoothinginterpolation, SI) image of figure 9 (b). Figure 9 (a), the cubic interpolated raw image, showed the "before restoration image' at twice the size and without the effects of SI. 


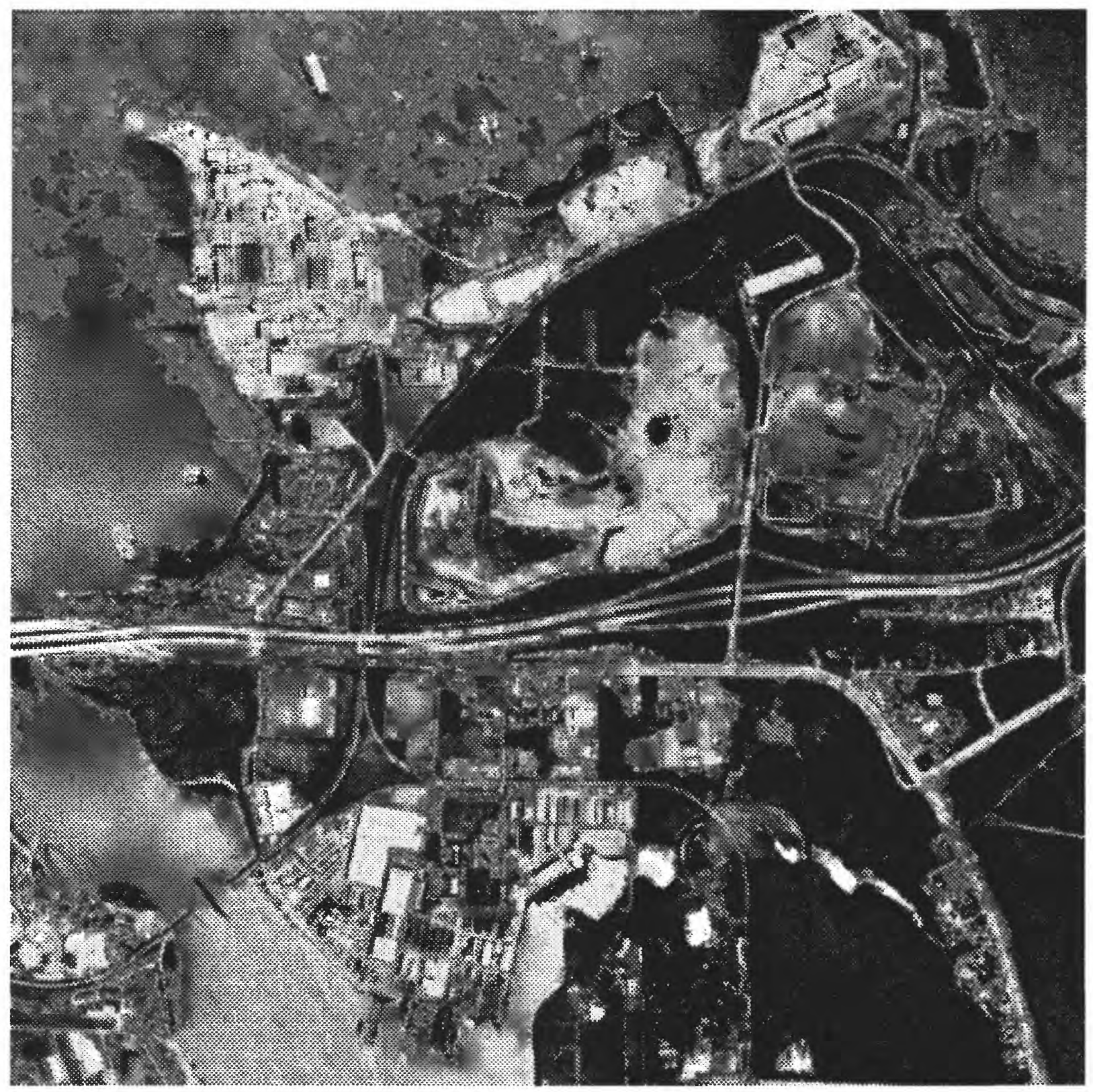

Figure 10 (b). Aerial photograph image at 5-m ground sample distance and enhanced by histogram equalization to improve the illustration. Cubic interpolated from the green color separate of a 4/10/88 NAPP photograph, (Dickson, 1989) scanned at 2-m sample distance, it is shown for comparison with the previous examples. 

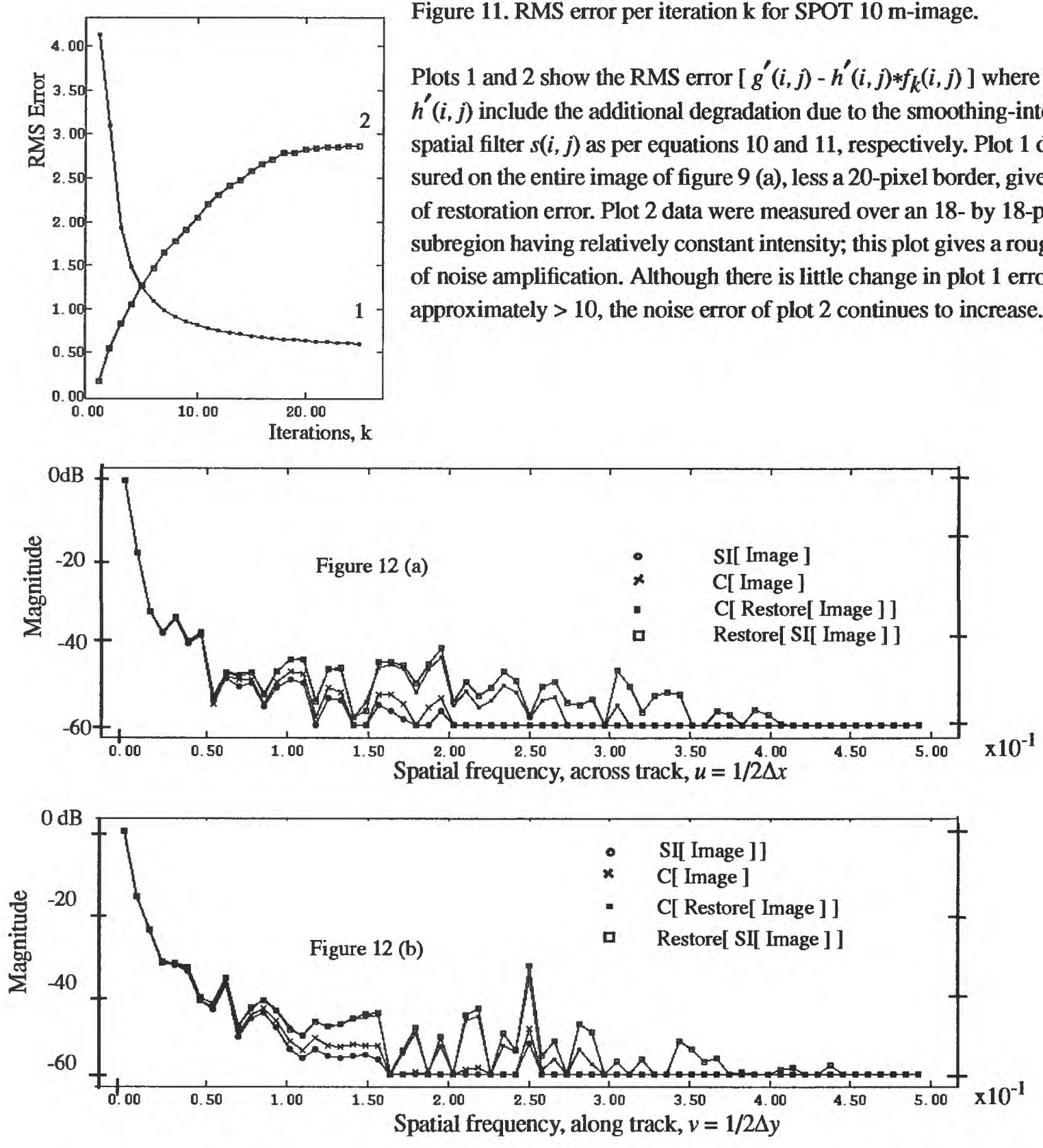

Figure 12. Fourier transforms of the SPOT image before and after restoration.

These plots are from the two-dimensional transform data shown in figure 13. All log magnitude discrete Fourier transform data were derived from the upper left quadrant ( $255 \times 256$ pixels) of the two-times enlarged SPOT 10-m images of figures 8(c), 9 (a), (b), or 10(a). In figure 12 (a) or (b), $\Delta x$ and $\Delta y$ are the normalized (1) sample distances of the enlarged images and thus spatial frequency 0.25 corresponds to the Nyquist frequency of the original size, raw image of figure 8(a). Restore[ Image ] and Restore[ SI [ Image ] ] denote, respectively, restoration of the original size image, 8 iterations, and restoration by smoothing-interpolation (SI) of the enlarged image, 13 iterations; C denotes cubic interpolation to twice the size. These conditions are shown as the upper two plots in (a) or (b). In each case, the result is an increase in the spatial frequency amplitudes of the original image; that is, where $u$ (or $v) \leq 0.25$. Spectra for $u$ (or v) $>0.25$ are believed to be due, in part, to interpolation and (or) noise effects and are the subject of further study. 


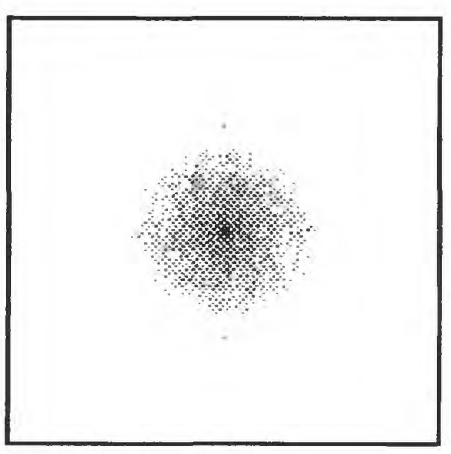

a

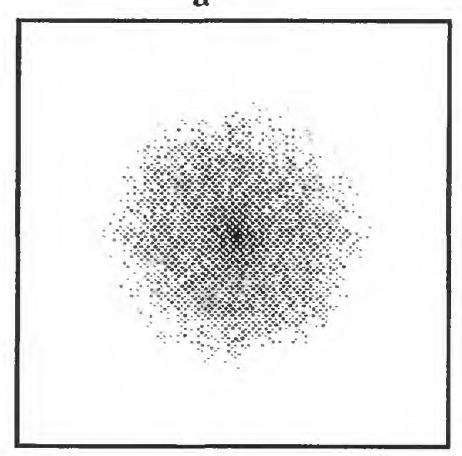

c

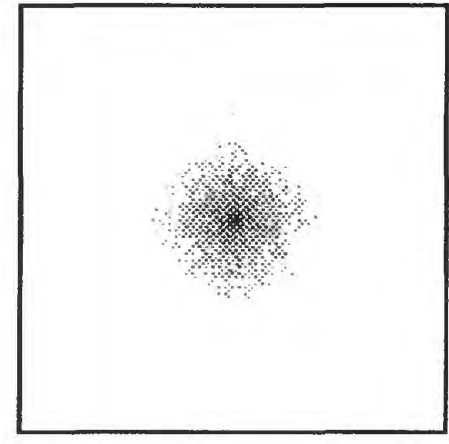

b

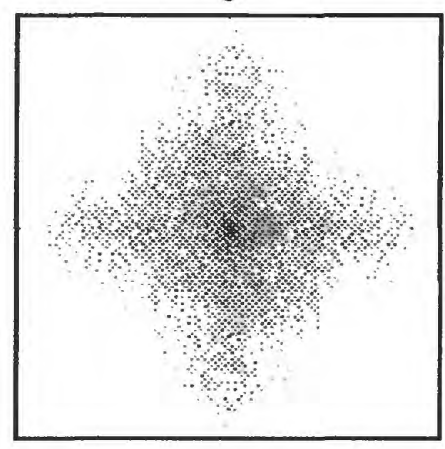

d (e) Orientation of spatial frequency axes

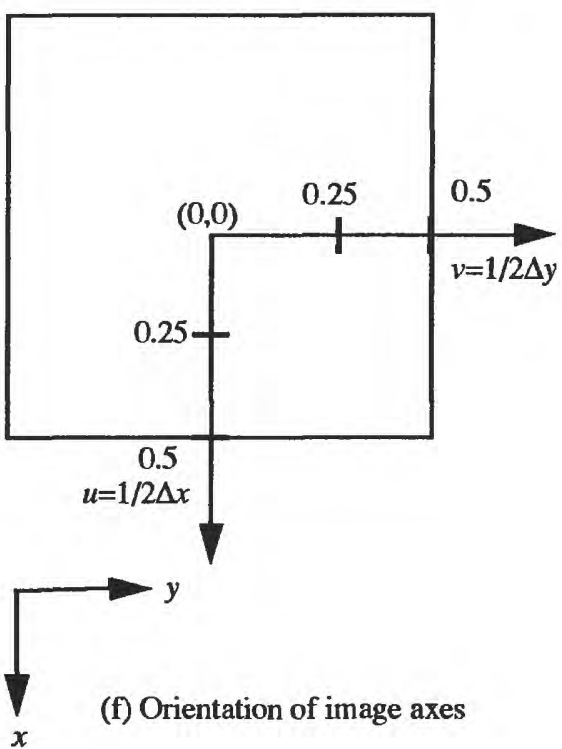

Figure 13. Fourier transforms of sample images, before and after restoration.

Profiles from these data along axes $(u, 0)$ and $(v, 0)$ were plotted in figure 12. For the log magnitude data of figure 13 , $0 \mathrm{~dB},-30 \mathrm{~dB}$ and $-60 \mathrm{~dB}$ are shown as black, mid-gray, and white, respectively. Test conditions are given in table 3; C and SI denote interpolating the SPOT image to twice its size by cubic or smoothing-interpolation, respectively.

Table 3

\begin{tabular}{|c|l|c|}
\hline Figure & \multicolumn{1}{|c|}{ Processing } & Iterations \\
\hline \hline $\mathrm{a}$ & C[ Image ] & none \\
\hline $\mathrm{b}$ & SI[ Image ] & none \\
\hline $\mathrm{c}$ & C[Restore[ Image ]] & 8 \\
\hline $\mathrm{d}$ & Restore[ SI[ Image]] & 13 \\
\hline
\end{tabular}

